\title{
Studying astrophysical reactions with low-energy RI beams at CRIB
}

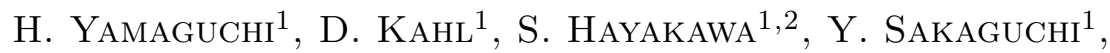 Y. Wakabayashi ${ }^{3,1}$, T. Hashimoto ${ }^{4,1}$, S. Cherubini $^{2,5}$, M. Gulino ${ }^{2,6}$, C. Spitaleri ${ }^{2,5}$, G.G. Rapisarda ${ }^{2,5}$, M. La Cognata ${ }^{2}$, L. Lamia ${ }^{5}$, S. Romano ${ }^{2,5}$, S. Kubono ${ }^{3,1}, \mathrm{~N}_{\text {IWASA }}{ }^{7}$, T. Teranishi $^{8}$,

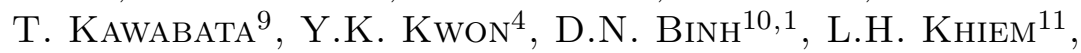
N.N. Duy ${ }^{12}$, S. Kato ${ }^{13}$, T. Komatsubara ${ }^{4}$, A. Coc ${ }^{14}$, N. DE

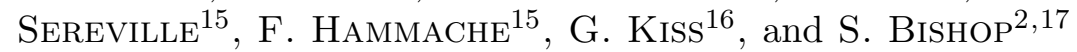

1 Center for Nuclear Study (CNS), University of Tokyo, Tokio, Japan

2 INFN - Laboratori Nazionali del Sud, Catania, Italy

3 RIKEN Nishina Center, Wako, Saitama, Japan

4 Institute for Basic Science, Daejeon, Korea

5 Dipartimento di Fisica e Astronomia, Università di Catania, Catania,

$$
6 \text { Università "Kore", Enna, Italy }
$$

7 Department of Physics, Tohoku University, Tohoku, Japan

8 Department of Physics, Kyushu University, Kyushu, Japan

9 Department of Physics, Kyoto University, Kyoto, Japan

$1030 \mathrm{MeV}$ Cyclotron Center, Tran Hung Dao Hospital, Hanoi, Vietnam

11 Institute of Physics, Vietnam Academy of Science and Technology,

$$
\text { Hanoi, Vietnam }
$$

12 Dong Nai University, Dang Nai, Vietnam

13 Department of Physics, Yamagata University, Yamagata, Japan

14 Centre de Spectrometrie Nucleaire et de Spectrometrie de Masse,

Orsay, France

15 Institut de Physique Nucleaire, Orsay, France

16 Institute for Nuclear Research (MTA-Atomiki), Debrecen, Hungary

17 Technische Universität München, München, Germany 


\begin{abstract}
Studies on nuclear astrophysics, nuclear structure, and other interests have been performed using the radioactive-isotope (RI) beams at the low-energy RI beam separator CRIB, operated by Center for $\mathrm{Nu}$ clear Study (CNS), the University of Tokyo. A typical measurement performed at CRIB is the elastic resonant scattering with the inverse kinematics. One recent experiment was on the $\alpha$ resonant scattering with ${ }^{7} \mathrm{Li}$ and ${ }^{7} \mathrm{Be}$ beams. This study is related to the astrophysical ${ }^{7} \mathrm{Li} /{ }^{7} \mathrm{Be}(\alpha, \gamma)$ reactions, important at hot $p$ - $p$ chain and $\nu$ p-process in supernovae. There have also been measurements based on other experimental methods. The first THM measurement using an RI beam has been performed at CRIB, to study the ${ }^{18} \mathrm{~F}(p, \alpha){ }^{15} \mathrm{O}$ reaction at astrophysical energies via the three body reaction ${ }^{2} \mathrm{H}\left({ }^{18} \mathrm{~F}, \alpha{ }^{15} \mathrm{O}\right) n$. The ${ }^{18} \mathrm{~F}(p, \alpha){ }^{15} \mathrm{O}$ reaction rate is crucial to understand the $511-\mathrm{keV}$ $\gamma$-ray production in nova explosion phenomena, and we successfully evaluated the reaction cross section at novae temperature and below experimentally for the first time.
\end{abstract}

\title{
1 Introduction
}

CRIB $[1,2]$ is a radioactive-isotope (RI) beam separator operated by Center for Nuclear Study (CNS), the University of Tokyo, installed at the RIBF facility of RIKEN Nishina Center. CRIB can produce low-energy $(<10$ $\mathrm{MeV} / \mathrm{u}$ ) RI beams by the in-flight technique, using primary heavy-ion beams accelerated at the AVF cyclotron of RIKEN $(\mathrm{K}=70)$. Most of the RI beams are produced via direct reactions such as $(p, n),(d, p)$ and $\left({ }^{3} \mathrm{He}, n\right)$, taking place at an 8-cm-long gas target with a maximum pressure of 760 Torr. A cryogenic target system, in which the target gas can be cooled down to about $90 \mathrm{~K}$, is currently available, and an intense ${ }^{7} \mathrm{Be}$ beam of $2 \times 10^{8}$ pps was produced using the system [3]. One main feature of the target system is the forced circulation of the target gas. We have found that the circulation of the target gas at a rate of 55 standard liters per minute (slm) was effective in eliminating the density reduction, caused by heat deposition of the beam. Solid targets such as beryllium foils can also be used as the production target. The secondary beam is purified with a magnetic analysis using dipole magnets, and with a Wien filter, which separates the beams according to their velocities. For relatively light RI beams such as ${ }^{7} \mathrm{Be}$, we obtained a purity close to $100 \%$ after the Wien filter. The Wien filter is operated with high voltages of $\pm 50-100 \mathrm{kV}$, supplied for a pair of 1.5 -m long electrodes with a gap of $8 \mathrm{~cm}$. For a stable operation at a higher voltage, we are making improvements on the insulators and other parts of the system. 
A list of typical parameters of RI beams produced at CRIB is found in [4].

The low-energy RI beams at CRIB are particularly suitable for studies on astrophysical reactions and nuclear resonant structure. An experimental method extensively used is the thick-target method in inverse kinematics [5]. In that method, the beam energy is degraded in a thick reaction target, and reactions occur at various center-of-mass energies. We detect light particles emitted after reactions, and reconstruct the kinematics. This method has several advantages, namely, (a) using inverse kinematics, we can study reactions with short-lived RI which cannot be used as the target, (b) we can perform simultaneous measurements of cross sections at various excitation energies without varying the incoming RI beam energy, and (c) when the beam is stopped in the target, we can perform measurements at $180^{\circ}$ in center-of-mass angle, where the Coulomb scattering is minimal. Many fruitful results have been obtained at CRIB with this method [6-16].

A recent major topic of our interest is the measurement of alpha-induced reactions. Several $(\alpha, p)$ reactions, such as ${ }^{14} \mathrm{O}(\alpha, p)[17],{ }^{11} \mathrm{C}(\alpha, p),{ }^{21} \mathrm{Na}(\alpha$, $p),{ }^{18} \mathrm{Ne}(\alpha, p),{ }^{30} \mathrm{~S}(\alpha, p)$, and ${ }^{22} \mathrm{Mg}(\alpha, p)$, have been studied at CRIB. For some of the recent measurements, an active target, referred to as "GEMMSTPC" [18], has been used. Measurements on the elastic resonant scatterings of a helium target and heavy-ion beams such as ${ }^{14} \mathrm{O},{ }^{21} \mathrm{Na},{ }^{7} \mathrm{Li}[12]$ and ${ }^{7}$ Be have also been performed. These measurements provide information on astrophysical $(\alpha, \gamma)$ reaction rates, and are also very suitable for studying nuclear cluster structure of the compound nuclei.

There are other projects for the determination of the astrophysical reactions using indirect methods with RI beams. The indirect measurement of the ${ }^{12} \mathrm{~N}(\mathrm{p}, \gamma)$ reaction, which is a key reaction to synthesize nuclei heavier than carbon, was performed by measuring ${ }^{12} \mathrm{~N}(d, n)$ reaction in inverse kinematics. Using the asymptotic normalization coefficient (ANC) method, the reaction rate was reevaluated [19]. We have performed an indirect reaction measurement using the Trojan horse method (THM) [20-22], as discussed later.

\section{$2 \quad{ }^{7} \mathrm{Be}+\alpha$ elastic resonant scattering}

A typical example of the resonant scattering experiment is the one on the ${ }^{7} \mathrm{Be}+\alpha$ scattering measurement. The measurement of the ${ }^{7} \mathrm{Be}+\alpha$ scattering allows us to evaluate the rate of the ${ }^{7} \operatorname{Be}(\alpha, \gamma)$ reaction, which is considered to play an important role in the hot $p-p$ chain and related reaction sequences [23]. Several reaction sequences including the ${ }^{7} \operatorname{Be}(\alpha, \gamma)$ reac- 
tion should take place in some high-temperature environments at $T_{9}>0.2$, where $T_{9}$ is the temperature in GK. In the $\nu p$-process in core-collapse supernovae [24], the ${ }^{7} \operatorname{Be}(\alpha, \gamma)$ reaction may contribute as much as the triple- $\alpha$ process to the synthesis of elements heavier than boron at the relevant temperature of $T_{9}=1.5-3$, according to a theoretical calculation [25]. The Gamow energy window at the highest temperature $T_{9}=3$ corresponds to the excitation energy $E_{\text {ex }}=8.2-9.6 \mathrm{MeV}$ in ${ }^{11} \mathrm{C}$. By our study, the resonant reaction rate should be evaluated more precisely by determining $\alpha$ widths for the resonances at high temperatures.

In 2010, we performed the measurement of the ${ }^{7} \mathrm{Be}+\alpha$ resonant elastic and inelastic scatterings with the thick-target method in inverse kinematics at CRIB [26]. A low-energy ${ }^{7} \mathrm{Be}$ beam at $14.7 \mathrm{MeV}$ was produced using a $2.3-\mathrm{mg} / \mathrm{cm}^{2}$-thick hydrogen gas target and a primary ${ }^{7} \mathrm{Li}$ beam at 5.0 $\mathrm{MeV} / \mathrm{u}$. The typical ${ }^{7} \mathrm{Be}$ beam intensity used in the measurement was 1-2 $\times 10^{5}$ per second at the secondary target, and the main measurement using a thick helium-gas target was performed for 4 days, injecting $2.9 \times 10^{10}{ }^{7} \mathrm{Be}$ particles into the target.

correspond to the resonance structure in ${ }^{11} \mathrm{C}$, We obtained an excitation function of the elastic scattering with several peaks, corresponding to the resonance structure in ${ }^{11} \mathrm{C}$. The obtained excitation is shown in the left panel of Figure 1. cross section. The curve is by an R-matrix calculation. An Rmatrix analysis was performed to deduce the parameters of the resonances, as the calculated curve also shown in the figure. A similar measurement was independently carried out by M. Freer et al. at other facilities [27], but our measurement included $\gamma$-ray detection to identify inelastic scattering events, and several differences were found in the obtained spectra [26].

The resonances observed in the present work might contribute to the astrophysical ${ }^{7} \mathrm{Be}(\alpha, \gamma){ }^{11} \mathrm{C}$ reaction rate at high temperature, $T_{9}>1.5$. We calculated the resonant reaction rate and compare it with the total reaction rate evaluated in NACRE $[28,29]$. In the evaluation reported in NACRE, only 2 resonances at 8.1045 and $8.420 \mathrm{MeV}$ are included. These two resonances dominate the reaction rate $N_{\mathrm{A}}\langle\sigma v\rangle$ up to the temperature $T_{9} \sim 3$, and a Hauser-Feshbach calculation was used to provide the reaction rate at higher temperatures. The resonant reaction rates were calculated for three resonances using analytical formula described in [28], and plotted in Fig. 1.

$\mathrm{r}$ resonances, but none of In conclusion, the resonances at $8.90 \mathrm{MeV}$ and $9.20 \mathrm{MeV}$ have a possibility to give significant contributions to the reaction rate for $T_{9}=1.5-3$, although they are unlikely to be more than the contribution of the $8.420-\mathrm{MeV}$ resonance, which dominates the reaction 

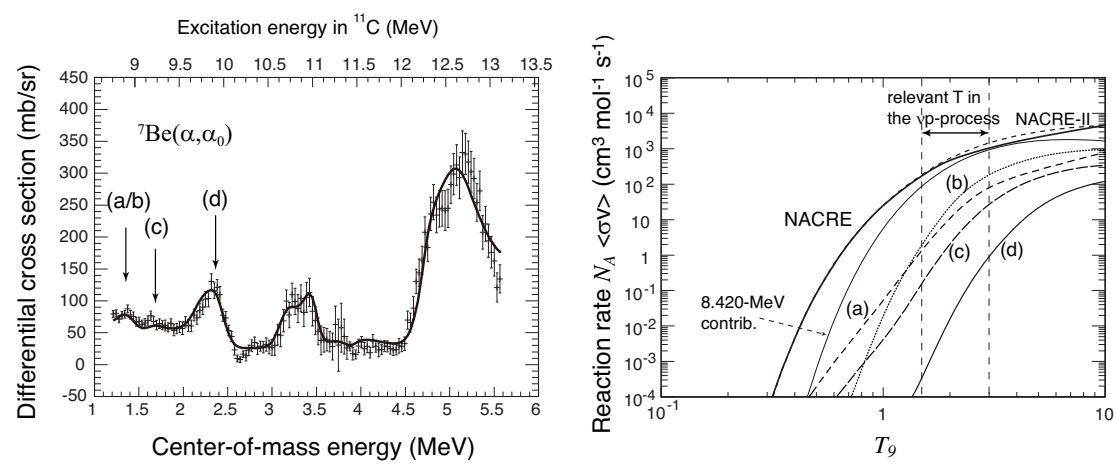

Figure 1: Excitation function of ${ }^{7} \mathrm{Be}+\alpha$ elastic scattering with an $\mathrm{R}$-matrix fit curve (left panel) and resonant reaction rate of ${ }^{7} \operatorname{Be}(\alpha, \gamma)$ for the 8.90, 9.20, and 9.97$\mathrm{MeV}$ resonances, calculated by the analytical formula. The evaluation by NACRE and NACRE-II are shown for comparison. The contribution by the $8.420-\mathrm{MeV}$ resonance, included in NACRE, is also shown.

rate.

\section{Study with the Trojan horse method and an RI beam}

The first measurement using the THM with an RI beam has recently been performed at CRIB [30]. The measurement was to study the ${ }^{18} \mathrm{~F}(p, \alpha){ }^{15} \mathrm{O}$ reaction at low energies relevant to astrophysics via the three body reaction ${ }^{2} \mathrm{H}\left({ }^{18} \mathrm{~F}, \alpha^{15} \mathrm{O}\right) n$. The ${ }^{18} \mathrm{~F}(p, \alpha){ }^{15} \mathrm{O}$ reaction rate is particularly responsible for the 511-keV $\gamma$ ray emission in nova explosion phenomena. The relevant temperature in novae is corresponding to the Gamow energy of 100-400 keV. A direct measurement of the ${ }^{18} \mathrm{~F}(p, \alpha)$ reaction was recently performed at TRIUMF [31], reaching to thee energy as low as $250 \mathrm{keV}$, but the reaction rate below that energy has been totally unknown experimentally.

The measurement at CRIB was performed with a ${ }^{18} \mathrm{~F}$ RI beam, of which maximum intensity was $2 \times 10^{6}$ pps. $\mathrm{A} \mathrm{CD}_{2}$ target with a thickness of 150 $\mu \mathrm{g} / \mathrm{cm}^{2}$ was irradiated by the beam, and 3-body reaction events were detected by a silicon detector array, referred to as ASTRHO, with additional double-sided silicon strip detectors. The analysis was performed by following a standard scheme of the Trojan horse method. 3-body events were selected according to the kinematical relationship of the detected charged particles, and converted into a Q-value spectrum. The momentum distri- 


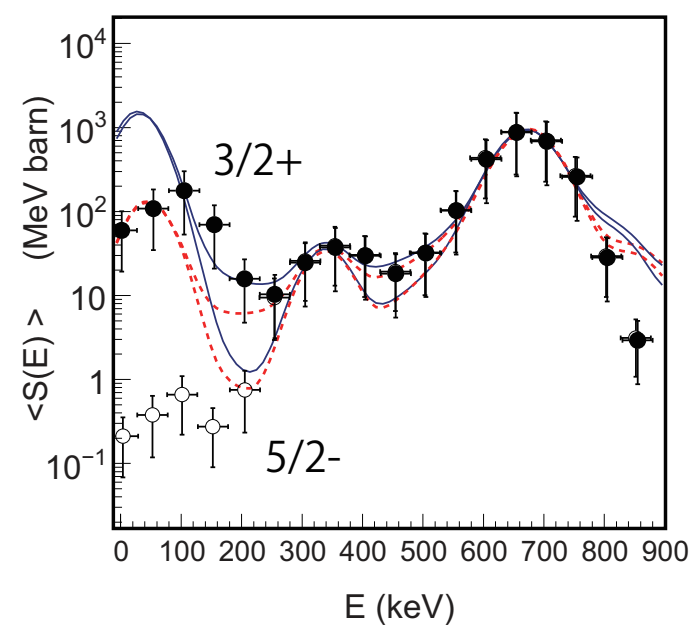

Figure 2: The astrophysical $S$-factor of the ${ }^{18} \mathrm{~F}(p, \alpha){ }^{15} \mathrm{O}$ reaction [30]. The filled and open circles show the $S$-factor obtained by the THM experiment at CRIB assuming $J^{\pi}=3 / 2^{+}$and $5 / 2^{-}$for the $6460-\mathrm{keV}$ resonance, respectively. The solid and dashed curves are calculations discussed in [31].

bution for the $p$ - $n$ intercluster motion was in agreement with a Hulthén function, which proves the dominance of the quasi-free mechanism. Then the three-body cross section was converted into the two-body cross section, using the momentum distribution of the $p$ - $n$ intercluster motion and a kinematical factor (see [30] for further details). We successfully evaluated the reaction cross section at the novae temperature and even below experimentally for the first time, as shown in Fig. 2. Because of the limited statistics due to the RI beam experiment, we could not uniquely determine the spin and parity $\left(J^{\pi}\right)$ of each level from the angular distribution. As a result, we had to assume $J^{\pi}$ for some of the resonances to obtain the total reaction cross section. The $S$-factor greatly deviated according to the $J^{\pi}$ assignment for the $6460-\mathrm{keV}$ resonance, which indicates the importance of the determination of $J^{\pi}$. Thereby we are planning to perform another measurement with greater statistics in 2015 .

\section{Acknowledgements}

The experiments were performed at RI Beam Factory operated by RIKEN Nishina Center and CNS, the University of Tokyo. We are grateful to the 
RIKEN and CNS accelerator staff for their help. This work was partly supported by JSPS KAKENHI (No. 21340053,25800125) from the Ministry of Education, Culture, Sports, Science and Technology (MEXT) of Japan.

\section{References}

[1] S. Kubono, et al., Eur. Phys. J. A13 (2002) 217.

[2] Y. Yanagisawa, et al., Nucl. Instrum. Meth. Phys. Res., Sect. A 539 (2005) 74-83.

[3] H. Yamaguchi, et al., Nucl. Instrum. Meth. Phys. Res., Sect. A 589 (2008) 150-156.

[4] D. Kahl, et al., AIP Conference Proceedings 1594 (2014) 163-170.

[5] K. P. Artemov, et al., Sov. J. Nucl. Phys 52 (1990) 408.

[6] M. Notani, et al., Nucl. Phys. A 746 (2004) 113c-117c.

[7] T. Teranishi, et al., Phys. Lett. B 556 (2003) 27-32.

[8] T. Teranishi, et al., Phys. Lett. B 650 (2007) 129-134.

[9] J. J. He, et al., Phys. Rev. C 76 (2007) 055802.

[10] H. Yamaguchi, et al., Phys. Lett. B 672 (2009) 230-234.

[11] J. J. He, et al., Phys. Rev. C 80 (2009) 015801.

[12] H. Yamaguchi, et al., Phys. Rev. C 83 (3) (2011) 034306.

[13] H. S. Jung, et al., Phys. Rev. C 85 (2012) 045802.

[14] J. J. He, et al., Phys. Rev. C 88 (2013) 012801.

[15] S. J. Jin, et al., Phys. Rev. C 88 (2013) 035801.

[16] L. Y. Zhang, et al., Phys. Rev. C 89 (2014) 015804.

[17] A. Kim, et al., Phys. Rev. C 92 (2015) 035801.

[18] T. Hashimoto, et al., Nuclear Instruments and Methods in Physics Research Section A 556 (1) (2006) 339 - 349.

[19] B. Guo, et al., Phys. Rev. C 87 (2013) 015803. 
[20] G. Baur, Physics Letters B 178 (2-3) (1986) 135-138.

[21] S. Cherubini, et al., Astrophys. J. 457 (1996) 855.

[22] C. Spitaleri, et al., Phys. Rev. C 69 (2004) 055806.

[23] M. Wiescher, et al., Astrophys. J. 343 (1989) 352-364.

[24] C. Fröhlich, et al., Astrophys. J. 637 (2006) 415.

[25] S. Wanajo, et al., Astrophys. J. 729 (2011) 46.

[26] H. Yamaguchi, et al., Phys. Rev. C 87 (3) (2013) 034303.

[27] M. Freer, et al., Phys. Rev. C 85 (2012) 014304.

[28] C. Angulo et al., Nucl. Phys. A 656 (1999) 3-187.

[29] Y. Xu, et al., Nucl. Phys. A 918 (2013) 61-169.

[30] S. Cherubini, et al., Phys. Rev. C 92 (2015) 015805.

[31] C. E. Beer, et al., Phys. Rev. C 83 (2011) 042801. 